\title{
EXPERIENCIAS DE COOPERACIÓN INTERUNIVERSITARIA MEDIANTE TIC: CONSORCIOS, REDES Y CAMPUS VIRTUALES COMPARTIDOS
}

\author{
(INTERUNIVERSITY COOPERATION EXPERIENCES USING ITC: PARTNERSHIPS, NETS AND \\ SHARED VIRTUAL CAMPUS)
}

\section{Jesús Salinas}

Universidad de las Islas Baleares (España)

\section{RESUMEN}

La utilización de las redes al servicio del aprendizaje en experiencias de aprendizaje mediante proyectos asumidos por consorcios o por redes de instituciones, ofrece un doble efecto: mejora y aumenta el acceso a las experiencias y materiales de aprendizaje a personas que tienen dificultades para el acceso a acciones convencionales, y contribuye a una actualización y al desarrollo profesional del profesorado que participa en la experiencia.

La colaboración puede tomar diversas formas, desde la simple distribución de materiales a la cooperación en proyectos que, en forma de asociaciones, consorcios, campus virtuales compartidos o redes institucionales, lleva al establecimiento de redes estables de aprendizaje.

A partir de experiencias diversas en este ámbito, se pretende ofrecer reflexiones y elementos de análisis, dado que los proyectos interinstitucionales que están surgiendo requieren de planteamientos claros y de una necesaria reflexión sobre estos mismos aspectos.

Palabras clave: entorno virtual, innovación, campus virtual compartido, red de aprendizaje, cooperación.

\begin{abstract}
The use of the learning networks to the formation in experiences of projects assumed by partnerships or networks of institutions, offers a double effect: improvement and increases to the access to the experiences and materials of learning to people who have difficulties for the access to conventional operations and contributes to the professional update and development of the teaching staff who participates in the experience.
\end{abstract}


The collaboration can be, from the simple distribution of materials to the cooperation in projects that, in form of associations, partnerships, virtual campuses shared or institutional networks, takes to the establishment of stable networks of learning.

From diverse experiences in this scope, it is tried to offer reflections and analysis elements, since the interinstitutional projects that are arising require of clear expositions and a necessary reflection on these same aspects.

Key words: virtual environment, innovation, shared virtual campus, learning network, cooperation.

\section{LA EDUCACIÓN SUPERIOR ANTE LA INTERNACIONALIZACIÓN Y LA GLOBALIZACIÓN}

No parece necesario argumentar a estas alturas que la evolución de la sociedad de la información y la emergente economía del conocimiento, impulsan nuevas y mayores demandas de cualificación, ni que a mayor formación de los individuos y de las sociedades se presentan mejores perspectivas de desarrollo.

En este contexto, la educación superior se está constituyendo cada vez más como un fenómeno global, que transciende las barreras institucionales, supera los límites de los campus o las mismas fronteras nacionales, impulsado manera decisiva por las TIC.

Para responder a los desafíos que plantea la sociedad del conocimiento, las universidades necesitan involucrarse en procesos de innovación docente apoyada en las TIC. Y, en el conjunto de todos estos procesos de innovación, los que suponen colaboración interinstitucional ofrecen, sin duda, interesantes perspectivas y desafíos.

Tanto en las sociedades avanzadas, como en las llamadas en vías de desarrollo se ha producido una afluencia de estudiantes a las universidades. Esta afluencia se ve acrecentada por la aplicación de las tecnologías de red a distintas modalidades de formación que diversifican la educación a distancia dando lugar a una flexibilización de los procesos de enseñanza-aprendizaje.

Una mirada a estos fenómenos desde la perspectiva iberoamericana, nos obliga a analizar la situación desde los fenómenos de la internacionalización y de la globalización. Ambos fenómenos constituyen aspectos centrales a la hora de abordar el tema de la cooperación interuniversitaria, dadas las complejas relaciones que se establecen entre la academia y las sociedades, entre lo nacional y lo global,... 
La cooperación interuniversitaria en el ámbito iberoamericano debe analizarse en el contexto la internacionalización de la educación superior de las últimas décadas, ya que constituye un asunto recurrente al hablar de investigación, desarrollo del conocimiento, acceso a este conocimiento por parte de investigadores, estudiantes y por los ciudadanos, formación, desarrollo personal y profesional, etc. Para el caso que nos ocupa, presenta dos aspectos centrales en relación con la cooperación y el uso de las TIC de lo que es necesario ocuparse: la movilidad de los estudiantes y los proyectos conjuntos.

Tanto movilidad como proyectos conjuntos, implantación de campus fuera de las fronteras, etc. que caracterizan los efectos de la globalización en la educación superior, reproducen algunas de las tendencias perversas de la globalización en otros ámbitos: dependencia de unos países que predominan, implantación de instituciones de países desarrollados, etc.

La evolución de las tecnologías de la información y, sobre todo, el desarrollo de Internet, la nueva economía basada en el conocimiento, etc... han provocado que los proyectos de internacionalización a que hacemos referencia no puedan entenderse sin enmarcarlos en la globalización...

Es necesario, por lo tanto, atender a ambos fenómenos: globalización e internacionalización. El primero lo entenderíamos como las tendencias de la educación que tienen implicaciones transnacionales: masificación de las universidades, mercado global para los estudiantes, profesiones y personal altamente especializado, alcance global de las TIC, inclusión y exclusión digital, etc.; mientras que la internacionalización hace referencia a las iniciativas y políticas de países y/o instituciones para abordar estas tendencias globales (política respecto al reclutamiento de estudiantes foráneos; colaboración con otras instituciones; establecimientos de extensiones de campus en otros lugares; etc.).

En la actualidad la internacionalización presenta nuevas facetas por el desarrollo de las TIC (entornos virtuales, campus virtuales, e-learning,...) que permiten la movilidad virtual de estudiantes, extensiones de campus fuera de las fronteras, proyectos suprainstitucionales de formación on-line, campus virtuales de distinta naturaleza entre los que entendemos como clave los campus virtuales compartidos, etc. regidos todo ellos en muchos casos por las reglas de la globalización.

Sus efectos son patentes: unos pocos países dominan el sistema científico global; las tecnologías de red son propiedad de corporaciones multinacionales 
y/o instituciones académicas de los países industrializados; el dominio del inglés (utilización del inglés como medio de instrucción e investigación) crea ventajas para determinados países; etc... En conjunto, representan riesgos que las instituciones de educación superior identifican como las principales amenazas (IAU, 2005): incremento de la comercialización del conocimiento y de los programas de formación, el incremento de programas de formación rápida para el puesto de empleo, y la fuga de cerebros.

Algunos datos pueden ayudar a comprender estos fenómenos:

- En 2006, la UNESCO estima en 2.5 millones de estudiantes en el nivel terciario siguiendo sus estudios en instituciones de fuera de su propio país (IIE, 2006). En estados Unidos, que es el país líder en la recepción de este tipo de estudiantes, en el curso 2005-06 esto supone 564.766 estudiantes extranjeros, de los que la mayoría son sufragados por sus propias familias, lo que supone una contribución neta a su economía por parte de dichos estudiantes y sus familias de 13.491 millones de dólares en concepto de matrículas y gastos básicos (IIE, 2007).

- Las perspectivas de futuro indican un crecimiento del fenómeno. Puede darnos una idea de su envergadura el informe de IDP Education Australia que predice que en 2025 al menos 8 millones de estudiantes seguirán programas transnacionales (IIE, 2006).

- La evolución de las sociedades hace que en estos momentos en países latinoamericanos, Asia o Europa, haya más demanda que oferta de plazas universitarias. Ahora muchos jóvenes salen de la educación secundaria y no encuentran plaza en las universidades de sus países, las cuales, por otra parte, no ven con buenos ojos la competencia de las lucrativas empresas norteamericanas que dirigen universidades con ánimo de lucro, acuden a rellenar la laguna. Como señalaba en el 2003 Anna Kuchment en Newsweek, "controlados por empresarios, estos centros -más de 700- venden educación práctica para alumnos con mentalidad práctica, y con ello ganan buen dinero. Ahora se están desarrollando en el extranjero, creando las primeras multinacionales, en un sector que siempre ha mirado con sospecha la cuenta de resultados".

- La implantación de estas instituciones en los países en vías de desarrollo (sea presencial o virtualmente) constituye un enorme desafío para las instituciones locales. Sirva como ejemplo la compra de instituciones de educación superior, 
presenciales y virtuales, por el grupo Sylvan/æ an/Laureate. En 2004, LAUREATE operaba una red foránea, integrada por 15 campus presenciales en 12 países de América Latina (Chile, Costa Rica, México, Panamá, Perú), de Europa (Francia, España, Suiza, Gran Bretaña, Países Bajos) y de Asia (China y, temporalmente India) (con aproximadamente 150,ooo estudiantes) y por 4 campus virtuales.

- En esta misma línea puede interpretarse el análisis de Didou (2006) respecto a la dinámica de los proveedores internacionales de educación superior y la dificultad para medir su impacto, sobre todo, en lo relativo a la educación a distancia y a través de Internet. A finales de los 90, una situación inédita modificó la reflexión sobre la internacionalización de la educación superior. Se trató de la aparición de proveedores transnacionales de educación superior, los cuales prestaban sus servicios conforme con esquemas comerciales de consumo. Con algunas excepciones, los proveedores transnacionales se instalaron en América Latina en los 90 y hacia finales de esta década y principios de la actual es cuando su consolidación se volvió un tema de indagación y, en menor medida, una cuestión política. Como señala Didou (2006, p. 22) "Hasta años recientes, las políticas de internacionalización de la educación superior han apuntado esencialmente a programas de movilidad académica y estudiantil y de constitución de redes, sin vínculos claros con los procesos de reforma educativa ni con la resolución de problemáticas propias. En paralelo, el fortalecimiento de proveedores transnacionales, que suministran servicios educativos con fines de lucro, también ha planteado retos inéditos".

Podemos analizar muchos más ejemplos, pero lo que parece claro es que las universidades no tienen ya el monopolio del saber, y, por tanto, disponen de dos opciones: o colaboran con otras instituciones y organismos de formación públicos y privadosy con las empresas que desarrollan herramientas de difusión del conocimiento y con empresas de informática y de telecomunicaciones, o tienen que competir en el mercado. Las tendencias en educación flexible y a distancia así parecen mostrarlo. Se presentan en este terreno dificultades a las universidades convencionales en relación a la capacidad de flexibilización de sus estructuras. Mantener el status de 'universidad tradicional' en un mundo de universidades transnacionales las puede convertir en no competitivas. Pero la especificidad a que hace referencia Bricall (1997) trae consigo tensiones en las relaciones entre las universidades. Tensiones que se manifiestan en dos direcciones: en la competencia y en la cooperación. 
La primera tensión, la competencia, constituye un elemento muy importante y que empuja a las universidades a la diferenciación -no todas las universidades deben ser iguales ni todas en su interior deben hacer lo mismo-. La universidad que lo hace todo y que es autosuficiente es básicamente un asunto de la Historia. La universidad debe escoger un área especial en la que tiene un dominio más decisivo, no únicamente desde el punto de vista de las materias, quizás desde el punto de vista de su entorno. A estas alturas, nuestro mundo académico está identificando todavía qué va a hacer y cuáles van a ser sus socios, está valorando el riesgo que corre compitiendo con los otros prestatarios de servicios educativos y formativos, sobre todo en lo que se refiere a experiencias de enseñanza a distancia basadas en la red. Las universidades que están intentando situarse en el mercado de la educación y formación se están encontrando con múltiples dificultades (Salinas, 1999). Unas son de orden técnico, porque las redes de telecomunicación no alcanzan, como muchos autores ya han señalado, en todas partes el mismo nivel de desarrollo. Otras apuntan a la capacidad de innovación de la organización (cambios administrativos, nuevas estructuras, implantación de nuevos servicios, flexibilidad del profesorado, etc.). Por otro lado, los mismos agentes competidores en el mercado de la formación, además de compartir el mercado, pueden descapitalizar a las universidades al arrebatarles (o compartir) uno de los bienes más preciados: sus profesores. En efecto, las universidades convencionales poseen profesores formados en los que se ha invertido algo que los competidores no tienen: tiempo. Compartir o ceder estos profesionales no parece ser una buena estrategia (Salinas, 1999).

En cuanto a la segunda tensión: la cooperación, las universidades tienen que trabajar con otras universidades o con otros grupos. Se puede rechazar la cooperación pero se va a encontrar con la presencia del mercado. Puede comprenderse que el éxito de las experiencias a desarrollar en las universidades convencionales dependerá de la transformación de algunas de las actuales estructuras que provocan el aislamiento institucional para potenciar equipos que conjuguen la calidad docente en sistemas presenciales con la interacción a través de las redes y que lleven a la cooperación en el diseño y la distribución de los cursos y materiales de educación a distancia en el marco de consorcios de instituciones dando lugar a verdaderas redes de aprendizaje, descritas en otros trabajos (Harasim y otros, 1995; Salinas, 1995, 1996, 2001).

Si admitimos la necesidad de profesionales cualificados y la necesidad de formación continua (fundamentalmente relacionada con los continuos cambios propiciados por la evolución tecnológica, la utilización, gestión y administración de la información, etc.) como elementos claves de esta era digital, la introducción de nuevas tecnologías en las universidades debe ser considerada medio privilegiado para 
alcanzar estos cambios (Salinas, 1999). Es indudable que asistiremos, y asistimos, a una inflación tanto de cursos on-line, como de experiencias de aprendizaje abierto, flexible, en cualquiera de las formas y grados que pueden surgir. Cualquier persona con una conexión a Internet puede apuntarse a los cientos de cursos de idiomas, mecanografía o informática que aparecen en la red. También en el terreno de la formación continua y superior se observa un incremento de las demandas de aprendizaje. Este incremento del mercado de formación lleva consigo, como hemos señalado, mayores oportunidades y mayor competitividad, tanto para las instituciones existentes, como para las de nueva creación. El reto aquí se encuentra en lograr el equilibrio entre la oportunidad y la calidad.

\section{LA COOPERACIÓN INTERUNIVERSITARIA Y LAS TIC}

La evolución constante de las telecomunicaciones y las tecnologías informáticas, junto a la progresiva comercialización que esta experimentando Internet, hacen que el ciberespacio vaya siendo dominado por grandes empresas de la comunicación audiovisual y del ocio y por instituciones comerciales de formación que se erigen en emisores privilegiados, concentrando el control sobre los contenidos y sobre las audiencias (segmentadas, individualizadas...).

Pero junto a esta organización, convive la comunicación horizontal, el intercambio, los emisores-receptores que vienen siguiendo la tradición de lo que a través de redes se ha venido haciendo. En la medida que puedan convivir ambos aspectos, la red, entendida como un servicio de intercambio horizontal, tendrá un gran potencial educativo, porque eso permitirá un gran flujo de comunicación institucional, personal, informal.

Desde esta opción pensamos sobre las redes en la educación, sobre las posibilidades de crecimiento de la intercomunicación, la comunicación horizontal, multidireccional que ha caracterizado a Internet, y lo hacemos considerando que constituyen verdaderos procesos de innovación educativa. Así, las redes de comunicación (aquéllas que operan superpuestas a las redes de telecomunicación), las redes formadas por personas que solicitan, proporcionan e intercambian experiencias e informaciones sobre un determinado de interés común a través de sistemas de comunicación, están configurando un nuevo contexto de comunicación que cada vez se universaliza y diversifica más y que, en el caso de su vertiente educativa, afecta a los distintos componentes del proceso de formación: los actores del proceso (profesor-mediador-animador-tutor, alumno-usuario de la formación), el modelo de formación y su sistema organizativo. 
Las transformaciones que presenciamos en el terreno tecnológico, en el económico y en el cultural, y que se concretan, entre otros, en cambios en la comunicación, afectan a las instituciones educativas, que tratan de responder a los distintos tipos de crisis que rodean a la enseñanza superior -consideramos válidos todavía los 3 tipos de crisis- que describe Daniel (1997): la crisis de acceso a la formación superior, cuando la demanda crece sin encontrar respuestas satisfactorias desde las instituciones convencionales, la crisis de costo, y la crisis de flexibilidad que conduce a reconsiderar la pertinencia de sus curricula y a enfrentarse a un cuerpo de estudiantes constantemente cambiante, incluyendo alumnos empleados más adultos, que están haciendo que las universidades se planteen cuestiones sobre cómo enseñan, ya que para muchos de estos estudiantes las clases magistrales en el salón de clase con horario fijo no es el modo preferido de aprender, ni posiblemente el más adecuado.

Parece claro que todos estos aspectos están condicionando tanto las formas en las que las universidades manejan sus proyectos transnacionales, como el modo de estructurarse los proyectos de cooperación entre universidades. La reflexión se hace necesaria ya que puede ocurrir que, mientras muchas instituciones de educación superior se están centrando en las nuevas tecnologías de la comunicación para distribuir educación a distancia, están fracasando en detectar lo que se necesita para resolver los problemas reales concernientes a la formación inicial y en ejercicio de los profesionales.

Entre las instituciones universitarias se detecta una decidida apuesta por las TIC, al mismo tiempo que reciben una fuerte demanda de sistemas de enseñanzaaprendizaje más flexibles y accesibles, menos costosos y a los que pueda incorporarse cualquier ciudadano a lo largo de la vida. Para responder a estos desafíos las instituciones educativas deben revisar sus referentes actuales y promover experiencias innovadoras en el campo de los procesos de enseñanza-aprendizaje apoyados en las TIC (Salinas, 1998, 2002).

$\mathrm{El}$ acceso al sistema de aprendizaje a través de redes, convierte en relativamente irrelevante el lugar y el tiempo de acceso. Qué diferencia habría entre acceder desde el aula de la universidad o desde el hogar a los materiales de aprendizaje a través de redes? Quizá sea conveniente disponer de cursos y materiales de aprendizaje para un doble uso, de tal manera que tanto los alumnos que asisten a la institución puedan acceder a ellos en el aula, en el centro, como aquellos que no pueden asistir al centro, puedan acceder a esos mismos materiales a través de redes. Todo ello, puede comprenderse, supone nuevas perspectivas organizativas tanto a nivel institucional como a nivel interinstitucional y/o internacional de la educación superior. 
En otros trabajos (Salinas, 1997, 2004) atribuíamos a la enseñanza flexible algunas de las características que se han asociado al aprendizaje abierto, ya que lo importante en ambos casos es precisamente que flexibiliza algunos de los determinantes del aprendizaje. Estos determinantes pueden pertenecer a dos dimensiones distintas, pero igualmente importantes al considerar proyectos interinstitucionales de educación superior:

- Una, relacionada con los determinantes administrativos relativos al concepto de distancia: asistencia a un lugar predeterminado, tiempo y número de sesiones, ser enseñado en grupo por el profesor, las reglas de la organización. Se refiere, por tanto, al grado de libertad, o mejor de opcionalidad, de los estudiantes en el acceso, admisión, selección de cursos, y libertad en los determinantes temporales y espaciales, tales como accesibilidad (credenciales académicas previas, tiempo, localización física, determinantes financieros, características personales, responsabilidad social), flexibilidad (frecuencia de los periodos de admisión, ritmo de aprendizaje, servicios de apoyo opcionales), control del alumno sobre el contenido y la estructura, elección del sistema de distribución, y acreditación.

- Otra dimensión relacionada con los determinantes educativos: especificación de metas de aprendizaje ajustadas a las características de los alumnos, contenidos y secuencia de enseñanza, la estrategia para enseñar del profesor individual o de la organización, variedad y adecuación de medios, uso abierto de los recursos bajo la responsabilidad del alumno-usuario y eficacia didáctica en estos contextos, etc.

Tanto desde la perspectiva del usuario, como desde la del profesor y administrador de la institución educativa, ambas dimensiones debieran tenerse en cuenta al configurar ambientes instruccionales apoyados en TIC, ya que conducen a una oferta educativa más flexible, que sirve tanto para aquellos alumnos que siguen la enseñanza 'presencial', como aquellos que siguen la enseñanza a distancia o por cualquiera de las formulas mixtas. Y, ello requiere modelos pedagógicos nuevos y un fuerte apoyo de las TIC.

El que las decisiones sobre el aprendizaje recaigan en gran medida en el usuario de la formación, constituye una de las razones por las que el concepto de aprendizaje abierto/enseñanza flexible aparece más y más asociado con el uso de sistemas multimedia e interactivos. En muchos casos, esto se asocia a la flexibilización de algunos de los determinantes del primer tipo, pero un buen proyecto en este terreno 
debe atender sobre todo a los determinantes didácticos. Ello supone importantes cambios en la concepción y en la práctica de la enseñanza superior. Se ha de entender que los cambios que introducen las tecnologías de la comunicación en la enseñanza no sólo afectan a los medios puestos en juego, afectan a todos los elementos del proceso educativo: organización, alumno, curriculum, profesor... Y, entre ellos, afectan a la organización, al marco en el que se desarrolla la comunicación educativa. Aquí habría que recordar la importancia de atender al contexto del alumno, o mejor, a la diferencia de contextos culturales, etc. que presentan los alumnos cuando se trata de experiencias multinacionales.

En la dinámica de lo que ocurre en el conjunto de instituciones de educación superior, las universidades iberoamericanas han experimentado un cambio de cierta importancia en el conjunto del sistema educativo de la sociedad actual (Silvio, 2000; Salinas, 2001; Facundo, 2004):

- Desplazamiento de los procesos de formación desde los entornos convencionales a otros entornos.

- Demanda generalizada para que los estudiantes reciban las competencias necesarias para el aprendizaje continuo.

- Comercialización del conocimiento que genera simultáneamente oportunidades para nuevos mercados y competencias nuevas en el sector.

- Aparición de nuevas organizaciones de enseñanza que se constituyen como consorcios o redes de instituciones y cuyos sistemas de enseñanza se caracterizan por la modularidad y la interconexión.

- Internacionalización.

- $\quad$ etc...

Todo ello exige a las instituciones de educación superior una flexibilización de sus procedimientos y su estructura administrativa para adaptarse a nuevas modalidades de formación más acordes con las necesidades que esta nueva sociedad presenta. Al mismo, tiempo reclama políticas consecuentes para preservar igualdad de oportunidades, universalización del acceso a la educación, flujo de pensamiento global y calidad de la educación superior.

Todo ello nos está empujando a la creación de auténticas redes de aprendizaje donde los recursos propios y los de las otras instituciones de la red se pongan verdaderamente al servicio de los alumnos de todas las instituciones participantes. 
Y esto, no puede lograrse sin una verdadera colaboración que implica proyectos de cooperación interinstitucional, en los que la coproducción es necesaria, pero no resulta suficiente.

La situación actual exige de nuestras universidades que compartan sus recursos educativos utilizando aquellas tecnologías de telecomunicaciones disponibles en cada caso y cooperen en el diseño y la producción de nuevos materiales. Exigen, en definitiva, que se estructuren consorcios o redes de aprendizaje en las que las distintas instituciones interesadas se incorporen a verdaderos proyectos de cooperación. La cultura de la cooperación no es espontánea y, por ello, es una las tareas que urge trabajar en este terreno. Al final, se tratará de organizar verdaderas universidades globales que al mismo tiempo constituyan consorcios o redes institucionales, acogiendo así proyectos conjuntos de cooperación.

Es imprescindible que las instituciones de enseñanza superior se involucren en experiencias de explotación de las TIC en la docencia. Y en estas experiencias, junto al apoyo institucional se considera elemento crucial el convencimiento del profesorado como elemento clave del éxito. Ello requiere fuerte motivación (sensibilización, reconocimiento, incentivos) del profesorado que participa o susceptibles de participar en experiencias de utilización de las TIC en la docencia universitaria (Silvio, 2003; Facundo, 2004).

Se trata de considerar la integración de las TIC en las instituciones de enseñanza superior en el contexto de la evolución de la sociedad, del cambio social, etc. Se trata de atender a la oportunidad de 'mercado' en cuanto aparecen nuevas oportunidades para las universidades en acciones alternativas a las convencionales.

Se requiere reflexionar sobre la necesidad y urgencia de introducirse en este terreno por parte de las universidades ante la competencia que en el terreno de las 'enseñanzas virtuales' van apareciendo y sobre los peligros de la excesiva comercialización del conocimiento (Silvio, 2003; Didou, 2005, 2006). La fortaleza de las universidades en el terreno de las TIC en la docencia está, como se dijo, en su profesorado y en el conocimiento. Pero ninguna universidad es fuerte en todos los campos. Se ve imprescindible la alianza entre las instituciones, la formación de equipos interdisciplinares, interinstitucionales que abarquen todos los aspectos necesarios para la calidad de los programas (técnico, pedagógico, comunicativo, etc.). 
En este sentido, Hiltz (1998) señala que en el uso de la comunicación mediada por ordenador para la educación en cualquier tiempo - cualquier lugar existen dos modelos:

- Uno es el mercado de masas que emula los modos de distribución del aprendizaje a distancia convencional de una vía para enviar materiales a los estudiantes, recibiendo materiales de ejercicios o test individuales de los alumnos y proporcionando algún medio uno-a-uno de comunicación entre el alumno y el tutor. Las páginas web pueden reemplazar el vídeo por clases, y el e-mail puede reemplazar al correo tradicional en la correspondencia alumno-profesor. Pero básicamente es el mismo modelo pedagógico.

- Un modelo diferente es la utilización de la tecnología para tratar de crear el tipo de comunidad de aprendizaje que puede surgir de un buen seminario, donde los estudiantes aprenden con y de los otros, colaborativamente, y los miembros docentes estructuran los temas, proporcionan experiencia y trabajan cerca de los estudiantes que preparan sus proyectos de presentación al grupo. Se trata ni más ni menos que de buscar modelos radicales en la nomenclatura de Roberts, Romm y Jones (2000).

\section{COLABORACIÓN EN PROYECTOS DE EDUCACIÓN SUPERIOR: ASOCIACIONES, CONSORCIOS Y REDES}

Si nos centramos en los proyectos conjuntos, un aspecto importante a la hora de calibrar los proyectos de cooperación en el ámbito de la educación superior lo constituye en tipo de organización en el que se apoya el proyecto (asociaciones académicas, consorcios académicos, redes institucionales,...).

Uno de los factores que condicionan el desarrollo de proyectos de cooperación interuniversitaria mediante las TIC lo constituye en tipo de organización en el que se apoya el proyecto. Es obvio que los organismos internacionales promueven proyectos relacionados con las TIC en el que suelen involucrar a diversas instituciones (ATEI -Asociación de Televisión Educativa Iberoamericana-; ASIVE, la Asociación Internacional que organiza Virtual Educa; algunos programas de las Cumbres y otros muchos pueden constituir ejemplos de ello), pero al margen de estas iniciativas, puede hablarse de tres tipos de organización, cada uno de ellos ofreciendo distintas características que pueden proporcionar mayor o menor carga de institucionalización, agilizar o entorpecer el desarrollo de los mismos, afectar a la 
gestión conjunta, de modo que influyen en la orientación, viabilidad y en el modelo mismo del proyecto:

- Asociaciones académicas: Habitualmente suele tratarse de académicos -y en algunas ocasiones sus respectivas unidades organizativas- que aúnan esfuerzos con el objetivo común de desarrollo profesional. En muchos casos en el seno de estas asociaciones se promueven proyectos de investigación o de docencia, que en número creciente pueden ser considerados de educación a distancia y/o de uso de las tecnologías en la formación. Algunos ejemplos: Asociación de Educación a Distancia y Tecnologías Educativas (EDUTIC) de Argentina; Asociación para el Desarrollo de la Tecnología Educativa y Nuevas Tecnologías en la Educación (EDUTEC), presente en 12 países iberoamericanos; Asociación Iberoamericana de Educación Superior a Distancia (AIESAD); la Red Iberoamericana de Informática Educativa (RIBIE), formada por grupos que desarrollan y utilizan la informática para el proceso educativo; etc...

- Consorcios académicos: En este caso nos encontramos ante agrupaciones de unidades académicas que se juntan para ejecutar un contrato o un proyecto concreto. Este contrato o proyecto bien puede ser una actividad académica común, bien el desarrollo de un acuerdo de franquicia -que describiremos más adelante-. Como ejemplo: El Consorcio Red de Educación a Distancia (CREAD), cuya misión apunta al desarrollo de la Educación a Distancia a nivel interamericano, mediante la cooperación interinstitucional; o el Consorcio Iberoamericano para la Educación en Ciencia y Tecnología (ISTEC) que desarrolla proyectos en el ámbito del uso de las TIC en educación.

- Redes institucionales: Estas redes están formadas por instituciones académicas con un perfil común, que se unen para acometer diversos propósitos. En nuestro caso, las instituciones se unen ante necesidades y oportunidades comunes que plantean las oportunidades y amenazas del uso de las TIC en proyectos de formación, marcados por la globalización, la internacionalización o al menos la suprainstitucionalización. Los ejemplos son variados: El Grupo G10-Colombia conformado por la Universidad Nacional de Bogotá, Universidad de Los Andes, Pontificia Universidad Javeriana de Bogotá, Pontificia Universidad Javeriana de Cali, Universidad del Valle, Universidad de Antioquia, Universidad Pontificia Bolivariana, Universidad Eafit, Universidad Industrial de Santander y Universidad del Norte que tiene por objeto compartir experiencias docentes mediante entornos virtuales; y en la misma línea, el G9 de Universidades formado por las universidades españolas: Universidad de Cantabria, 
Universidad de Castilla La Mancha, Universidad de La Rioja, Universitat de les Illes Balears, Universidad de Oviedo, Universidad del País Vasco, Universidad Pública de Navarra, Universidad de Zaragoza y Universidad de Extremadura; la Red Universitaria de Educación a Distancia de Argentina (RUEDA); Red Universitaria Nacional de Chile (REUNA), uno de cuyos proyectos constituye la Universidad Virtual, etc.

Si observamos las distintas experiencias de uso de las TIC en proyectos interuniversitarios, podemos encontrar desde experiencias orientadas al mercado que se desarrollan enteramente como procesos de marketing, a verdaderos proyectos de colaboración. Bates (1999), describe tres modelos de educación a distancia internacional que pueden ayudarnos a clarificar este asunto:

- Educación a distancia que desarrolla un marketing directo. Unainstitución ofrece sus programas de educación a distancia de forma individual en distintos países. Se trata de la forma más común de captar estudiantes internacionalmente. Esto funciona mejor cuando el idioma es común y hay cierta semejanza cultural.

- 'Acuerdos de franquicia'. El programa es diseñado por una institución pero distribuido por otra, bajo un acuerdo de licencia o contrato. Entre las ventajas, puede destacarse, en primer lugar, que contribuye a reducir el riesgo financiero de la institución promotora. Segundo y quizá el más importante, la institución local puede proporcionar adaptación cultural tanto en la etapa de desarrollo como de distribución. El alumno puede participar así en su propio idioma. Finalmente, puede suceder que los alumnos de la institución que distribuye el programa puedan acceder a la institución promotora superando los sistemas rigurosos de entrada. Sin embargo, con un acuerdo de franquicia no hay igualdad entre los socios. Se asume que un socio tiene más experiencia o conocimiento que el otro. La ventaja para el socio 'débil' es que esta sociedad puede darle ventaja competitiva sobre otras instituciones en su jurisdicción.

- Programas conjuntos. Se trata de programas conjuntos entre dos o más instituciones en igualdad de condiciones, aunque tomando diferentes formas. Pueden acordar un curriculum conjunto y compartir la distribución del mismo, accediendo los estudiantes en igualdad de condiciones a los cursos de cada una de las instituciones sea de modo tradicional sea en modo en-línea.

Indudablemente, podemos encontrar multitud de experiencias de los tres tipos en el ámbito iberoamericano. Seguramente podemos vernos reflejados trabajando según uno o varios de estos modelos (Didou, 2006). 
Es indudable que, con lo dicho hasta ahora, estamos abogando por la colaboración interinstitucional en el ámbito de la utilización de las TIC y de la educación superior en general. Como hemos visto esta colaboración puede tomar diversas formas, desde la simple distribución de materiales a la cooperación en proyectos que, en forma de redes institucionales, lleva al establecimiento de redes estables de aprendizaje.

\section{REDES Y CONSORCIOS DE EDUCACIÓN SUPERIOR, HORIZONTES DE COLABORACIÓN}

Las redes de aprendizaje, las alianzas institucionales para explotar las posibilidades de las TIC en la educación, representan una alternativa organizada para el aprendizaje continuado.

Tal como decíamos anteriormente, los avances tecnológicos nos están empujando a la creación de auténticas redes de aprendizaje donde los recursos propios y los de las otras instituciones de la red se pongan verdaderamente al servicio de los alumnos de todas las instituciones participantes. Y esto, no puede lograrse sin una verdadera colaboración que implica proyectos de cooperación interinstitucional, en los que la coproducción es necesaria, pero no resulta suficiente.

La situación actual exige de nuestras universidades que compartan sus recursos educativos utilizando aquellas tecnologías de telecomunicaciones disponibles en cada caso y cooperen en el diseño y la producción de nuevos materiales. Exigen, en definitiva, que se estructuren consorcios o redes de aprendizaje en las que las distintas instituciones interesadas se incorporen a verdaderos proyectos de cooperación.

Buscando constituirse en un instrumento que permita a las instituciones participantes enfrentar un mundo altamente complejo -económica y políticamente-, cada vez más influido por el proceso de la globalización, el enfrentamiento de culturas y la necesidad de la protección de las identidades regionales y patrimoniales, se requieren iniciativas de colaboración internacional interuniversitaria.

Ninguna universidad permanece impasible ante el fenómeno que nos ocupa, y las respuestas son muy complejas, como no puede ser menos en unos contextos de diversidad tecnológica, cultural, de diversidad de las crisis a las que hay que enfrentarse, etc...

La situación actual exige de nuestras universidades que compartan sus recursos educativos utilizando aquellas tecnologías de telecomunicaciones disponibles en 
cada caso y cooperen en el diseño y la producción de nuevos materiales. Exigen, en definitiva, que se estructuren consorcios o redes de aprendizaje en las que las distintas instituciones interesadas se incorporen a verdaderos proyectos de cooperación.

Entre una coproducción reducida al aspecto financiero y una coproducción altruista, las instituciones universitarias deberíamos encontrar el equilibrio para la cooperación, para el desarrollo de proyectos de interés común aportando cada uno aquellos recursos -personales, técnicos y financieros- de que pueda disponerse para abordar proyectos dirigidos a la mejora de la educación superior (Salinas, 2001). Los avances tecnológicos nos están empujando a la creación de auténticas redes de aprendizaje donde los recursos propios y los de las otras instituciones de la red se pongan verdaderamente al servicio de los alumnos de todas las instituciones participantes. Y esto, no puede lograrse sin una verdadera colaboración que implica proyectos de cooperación interinstitucional, en los que la coproducción es necesaria, pero no resulta suficiente.

Los proyectos de utilización de entornos virtuales de formación, transformando las estructuras y organización de las instituciones de educación superior o haciendo aparecer nuevos tipos de organizaciones educativas, responden a variados modelos. A la vista de las variadas experiencias con TIC en la formación en línea, sobre todo en el nivel superior, puede comprobarse que ofrecen distintos grados de evolución, que en gran medida se traduce en nivel de complejidad y madurez del sistema.

Los resultados esperados ofrecen distinta fisonomía: centros de investigación conjuntos o complementarios, proyectos de investigación, programas de doctorado conjuntos o complementarios, intercambio de estudiantes de pregrado y postgrado, y proyectos culturales conjuntos, campus virtuales compartidos, etc...

Parece necesario una nueva estrategia en la 'política' referida a servicios y apoyo a los proyectos de integración de las TIC en cada una de las universidades de cara a reorganizar y racionalizar los servicios y estructuras de apoyo a la integración de las TIC en la docencia de cada universidad y de todas ellas en el conjunto de la red establecida. Las telecomunicaciones no son las que forman verdaderamente las redes. Para que exista una verdadera red, se necesita un entramado de personas, tecnología e instituciones, donde el elemento más importante lo constituye el factor humano desde el momento en que decide compartir recursos o cooperar en su creación. 
Son, al menos, dos grandes niveles de actuación los que se identifican en este proyecto:

- Nivel institucional, en el que se persigue el estudio y análisis de los procesos, normativas, estructuras, políticas, estrategias y reglamentos que las distintas universidades emplean a nivel funcional y de procesos (finanzas, personal, investigación, docencia, postgrado). Como consecuencia de ello se espera que las mejores prácticas implementadas por cada una de las instituciones puedan ser interiorizadas por las otras para generar una plataforma común que permita el trabajo conjunto en las áreas específicas que finalmente se decida compartir.

- Áreas específicas, a desarrollar en paralelo, y para lo cual es fundamental buscar el acercamiento entre grupos de investigadores, académicos y profesionales que identifiquen áreas disciplinarias en las que exista competencia, a nivel internacional, para elaborar y ejecutar trabajos conjuntos en investigación, pre y postgrado, y vinculación con el medio.

Así pues, una red de aprendizaje, tal como la establecida con este Campus Virtual compartido, la constituirían instituciones y/o personas que quieren promover un proyecto de formación en común en el que los usuarios-alumnos acceden a los materiales de aprendizaje independientemente de la institución de origen y puede establecerse una interacción entre los profesores y alumnos de las distintas instituciones.

La organización en esta situación resulta considerablemente afectada por la diversidad de usuarios de la red. Debemos dirigirnos a alumnos-usuarios de las mismas instituciones con mayor o menor grado de presencia en el campus, debemos considerar a los profesores que posiblemente se encuentren en las mismas condiciones, pero también hemos de tener en cuenta a posibles usuarios -alumnos y profesores- que no pertenezcan a las instituciones. Se han de considerar aspectos relacionados con la matrícula, la evaluación etc. en los centros remotos o en los más cercanos, etc.

Habría que determinar los servicios más adecuados a las actuaciones previstas. De esta forma, los alumnos-usuarios sean presenciales o no, participen desde un campus convencional o desde uno de los centros de aprendizaje o desde el propio hogar, pueden formar grupos de aprendizaje con estudiantes de otras universidades, compartir ideas y recursos, interaccionar con expertos, colaborar en la elaboración de proyectos comunes. 
Los profesores, por su parte, además de participar en estas experiencias, encuentran la oportunidad de interaccionar con otros profesores y compartir ideas. $\mathrm{Al}$ final se está ofreciendo la oportunidad para la interacción sobre cualquier tema, con colegas y expertos, la participación activa en la construcción del conocimiento y el intercambio de información.

Por las características de las distintas instituciones, por la naturaleza de los actores y de los cambios y transformaciones necesarios para llevarlo a cabo, este proyecto tiene que estudiarse como una innovación, como un proceso de cambios y mejoras, sistematizado, formalizado y que requiere seguimiento y evaluación.

Aún tratándose de una experiencia novedosa, los cambios que requiere en el proceso de enseñanza, en las organizaciones, etc. no son nuevos, ni tampoco su estudio. Disponemos de un sólido marco teórico, de estudios empíricos y aplicaciones prácticas. Todo ello suele encuadrarse en una serie de modelos de cambio que responden a grandes perspectivas y orientan los distintos estudios e investigaciones sobre la innovación. Partiendo de que la innovación es generalmente un proceso intencional y sistemático, como se ha venido afirmando a lo largo de este trabajo, pero que puede ocurrir de diversas maneras, numerosos autores han realizado cuidadosos análisis de experiencias de innovación ya ocurridas, identificando, a partir de estas, modelos del proceso de cambio.

\section{CAMPUS VIRTUALES COMPARTIDOS}

Podemos encontrar un tipo de proyectos de colaboración que pueden desarrollarse en el contexto nacional como en el internacional y que podemos denominar Campus Virtual compartido. Intercampus, por ejemplo, es un proyecto de las universidades públicas catalanas impulsado por el DURSI de la Generalitat de Cataluña cuyo objetivo principal es la creación de un modelo de organización de docencia compartida entre universidades que permita potenciar nuevos métodos pedagógicos y posibilitar nuevas formas de aprendizaje y de comunicación utilizando las TIC para crear un modelo de docencia a distancia y unificar procesos de gestión universitaria (Fandos). ADA-Madrid que aglutina las universidades públicas de la Comunidad Autónoma de Madrid presenta características parecidas. O el más reciente, Campus Andaluz Virtual. Otras experiencias como el Campus Virtual Compartido del G9 de universidades o el G-10 Colombia responden a proyectos de colaboración más horizontal. 
La puesta en marcha de las experiencias de Campus Virtuales compartidos no responden estrictamente a ninguno de los modelos propuestos por Hanna (1998). Realmente se trata de una alianza o consorcio de instituciones del sexto tipo (Universidades tradicionales extendidas) para organizar una estructura que respondería a la definición de Universidad Multinacional Global en algunos aspectos. Esto se da sobre todo en los casos del G-10 Colombia y el G9 de universidades españolas, como se ha indicado.

Así pues, el Campus Virtual compartido constituye en sí un nuevo modelo, o la aplicación de la cooperación que hemos descrito como programas conjuntos, para la puesta en marcha de una experiencia de formación en línea entre varias universidades que comparten docencia, alumnos e infraestructuras. En otras palabras, nos encontramos ante una experiencia donde varias instituciones en igualdad de condiciones acuerdan compartir la distribución del curriculum, accediendo los estudiantes en igualdad de condiciones a los cursos de cada una de las instituciones en modo en-línea.

Los avances de las TIC requieren del desarrollo de instituciones de educación superior adaptadas a una realidad en permanente evolución. Esto es, no sólo capaces de impulsar y liderar positivamente estas transformaciones globales, sino también, capaces de dar respuesta a las nuevas demandas que éstas generan en los ciudadanos.

Fijémonos en las universidades del G9 que han establecido un proyecto -Campus Virtual Compartido- de educación flexible con la idea de extender el campus universitario fuera del recinto tradicional, aprovechando las nuevas tecnologías para llegar a ciudadanos que de otra forma no tendrían acceso a una formación superior.

El establecimiento de asociaciones estratégicas orientadas a la colaboración interuniversitaria se presenta como un factor clave para avanzar hacia sistemas organizativos que superen las debilidades de concepciones caracterizadas por estructuras rígidas yjerárquicas obsoletas en un contexto en transformación constante en pro de estructuras más flexibles. Entre otros factores, por permitir minimizar los costes asociados al desarrollo tecnológico (dotación de recursos e infraestructuras, desarrollo de acciones de formación permanente del profesorado,...) maximizando sus beneficios (capacidad de liderar y gestionar el cambio tecnológico). O, dicho de otro modo, transformar los costes en inversiones rentables y rentabilizables como una de las líneas estratégicas de cada una de las instituciones y de todas en su conjunto. La generación de redes de conocimiento para la mejora tanto de la práctica diaria 
como de la calidad y la competitividad de las propias instituciones de formación superior aparece como solución en este contexto.

El CVC-G9 pretende, concretamente, ser un espacio donde las instituciones y también cualquier profesional de las universidades participantes comparta, intercambiey promueva proyectos relacionados con la explotación delas posibilidades educativas de las tecnologías de la comunicación, mediante:

- La ordenación de los proyectos ya en marcha (Asignaturas de libre configuración compartidas, Programa de profesores Invitados en Red, Formación compartida).

- El intercambio formal e informal de experiencias referidas al diseño, producción, uso y evaluación de proyectos, cursos y medios didácticos basados en el uso de las TIC en la enseñanza.

- El debate académico respecto a las tecnologías de la comunicación aplicadas a la educación.

- La organización de actividades apoyadas en las posibilidades comunicativas de las redes y la experimentación de herramientas de aprendizaje colaborativo.

- Proyectos de innovación por parte de grupos de profesores del colectivo, etc...

- Recursos didácticos, materiales de aprendizaje, etc. compartidos.

Como se ha dicho, el Campus Virtual compartido constituye una experiencia de aplicación de las TIC a la docencia donde se comparten recursos humanos y técnicos y se intercambian alumnos en un consorcio de universidades. Si se considera que la universidad ha de liderar socialmente la innovación del sistema educativo e investigador con objeto de proporcionar calidad a los servicios demandados por la sociedad, en estos momentos esto supone un planteamiento de futuro de las posibilidades del e-learning y de una adecuada utilización de las tecnologías de la comunicación en la docencia, al mismo tiempo que se requiere la organización de redes y consorcios potentes que compartan los recursos de aprendizaje necesarios en dichas experiencias. La experiencia del Campus Virtual Compartido del G9 de universidades, constituye una respuesta a este reto que podríamos caracterizar por:

- Surgir de la asociación de las distintas universidades con sus peculiaridades. Esto supone habitualmente dificultades, pero al mismo tiempo atender a cada una de estas peculiaridades nos lleva a desarrollar procedimientos de trabajo válidos para proyectos de cooperación de tipo horizontal, que pueden servir para 
la organización de otros tipos de consorcios, donde se considere importante respetar la diversidad de instituciones que los componen.

- $\quad$ Partir de propuestas realistas, es decir, no caer en el proyectismo. Las metas propuestas siempre han sido modestas y la metodología para su implantación permite un constante ajuste de los proyectos a las circunstancias reales. Todo ello supone una mayor garantía de éxito. Conocer la dimensión equilibrada del Campus Virtual compartido de forma que se mantenga dentro de los costos razonables y proporcione ventajas visibles a cada una de las universidades supone un reto de cara a mantener estas propuestas realistas.

- Preparar a las respectivas universidades para la oferta de títulos oficiales impartidos conjuntamente, considerando en cualquier caso las dificultades que esto encierra.

- Todos los proyectos iniciados lo son de cara a la mejora de la docencia, centrada en la explotación de las posibilidades que las TIC ofrecen en la enseñanza universitaria.

El éxito de estos proyectos dependerá de la transformación de algunas de las actuales estructuras, potenciando equipos que conjuguen la calidad docente en sistemas presenciales con la interacción a través de las redes y que lleven a la cooperación en el diseño y la distribución de los cursos y materiales de educación a distancia, en el marco de consorcios de instituciones dando lugar a verdaderas redes de aprendizaje. Y para que exista una verdadera red, se necesita, mucho más que telecomunicaciones, un entramado de personas, tecnología e instituciones, donde el elemento más importante lo constituye el factor humano desde el momento en que decide compartir recursos y experiencias o cooperar en su creación o compartir la docencia.

\section{REFLEXIONES FINALES}

Como apunte final y apoyándonos en lo que hemos venido diciendo, consideramos que debe reflexionarse y, en su caso, comenzar a tomar medidas sobre la urgente necesidad para las universidades iberoamericanas de cooperar con instituciones de distintos países interesadas en llevar a cabo experiencias en el campo de la formación para llegar a formar verdaderas redes de aprendizaje, verdaderas redes de cooperación. La cultura de la cooperación no es espontánea y, por ello, es una las tareas que urge poner en marcha en este terreno. Al final, se tratará de organizar 
verdaderas universidades globales que al mismo tiempo constituyan consorcios o redes institucionales, acogiendo así proyectos conjuntos de cooperación.

La utilización de las redes como instrumentos al servicio de la formación en experiencias de aprendizaje abierto, sean a cargo de una sola institución, sean mediante proyectos asumidos por consorcios o por redes de instituciones, ofrece un doble efecto: mejora y aumenta el acceso a las experiencias y materiales de aprendizaje a personas que tienen dificultades para el acceso a acciones convencionales, y contribuye a una actualización y al desarrollo profesional del profesorado que participa en la experiencia.

En este sentido, proponemos una serie -abierta- de elementos de reflexión:

- Los proyectos institucionales de cooperación horizontal:

- La cooperación entre países de la región como sistema para superar la dependencia y los efectos de la globalización.

- La importancia de una oferta contextualizada que se adapte a las necesidades concretas de cada comunidad (combinación de cooperación y especificidad de las universidades).

- Si pretendemos preparar a nuestra institución para el futuro, es importante involucrar a toda la comunidad universitaria, y esto en sus primeros momentos puede estar reñido con proyectos estrella. Se ha de tener claro que es lo que se pretende a medio y largo plazo. Es imprescindible que las instituciones de enseñanza superior se involucren en experiencias de explotación de las TIC en la docencia.

- Todo ello se ha de contemplar desde la perspectiva de proyectos de innovación educativa.

- Los cambios en las modalidades de formación y nuevas estrategias:

- $\quad$ No parece razonable seguir utilizando las estrategias clásicas de enseñanza en las distintas ramas de la enseñanza universitaria cuando se integran las TIC en la misma.

- Vemos la necesidad de nuevos modelos que ayuden a los profesores universitarios a afrontar la docencia explotando las posibilidades de 
estas tecnologías (utilización de lenguajes más icónicos, requerimientos distintos de presentación de la información, cambios en las funciones de los docentes, cambios en los modelos comunicativos, etc...).

- El crecimiento de la movilidad virtual conduce a la generalización de sistemas apoyados en los entornos virtuales de enseñanza-aprendizaje ya sea en proyectos de educación a distancia o en formatos más flexibles.

- Énfasis en la docencia, en el profesorado.

- La disponibilidad de la tecnología por parte de la institución no es el elemento crucial en este tipo de experiencias. El elemento clave es el profesorado. Por ello, el núcleo de las acciones que acompañan a un proyecto de utilización de las TIC en la enseñanza universitaria debe ir encaminado a la formación y al apoyo al profesorado.

- $\quad$ Puede surgir un peligro: los mismos agentes competidores en el mercado de la formación, además de compartir el mercado, pueden descapitalizar a las universidades al arrebatarles (o compartir) uno de los bienes más preciados: sus profesores.

- $\quad$ La necesidad de redes de aprendizaje.

- El intercambio de docencia en los grupos y redes de universidades estudiadas y en proyectos transinstitucionales de acciones formativas de forma flexible, continuada e intercambiable nos lleva a pensar que la cooperación en este ámbito de la explotación de las TIC en la docencia resulta imprescindible en un mundo de cambio para las universidades.

- Para que exista una verdadera red, se necesita, mucho más que telecomunicaciones, un entramado de personas, tecnología e instituciones, donde el elemento más importante lo constituye el factor humano desde el momento en que decide compartir recursos y experiencias o cooperar en su creación o compartir la docencia.

- Esto implica nuevas situaciones didácticas, nuevos entornos para el alumno-usuario, y por ello debemos contemplar una combinación de: trabajo autónomo; actividades presenciales (en el aula convencional o mediante videoconferencia); trabajo colaborativo en grupo; prácticas 
tutorizadas; tutoría telemática y/o con apoyo local, etc., para los que debemos preparar a nuestros alumnos y a nuestros profesores. Por ello la reflexión sobre este tipo de experiencias y las propuestas de mejora han de ser permanentes.

- La Oportunidad.

- $\quad$ Se trata de considerar la integración de las TIC en las instituciones de enseñanza superior en el contexto de la evolución de la sociedad, del cambio social, etc. Se trata de considerar la oportunidad de 'mercado' en cuanto aparecen nuevas oportunidades para las universidades en acciones alternativas a las convencionales.

- Se requiere reflexionar sobre la necesidad y urgencia de introducirse en este terreno por parte de las universidades ante la competencia que en el terreno de las 'enseñanzas virtuales' van apareciendo y sobre los peligros de la excesiva comercialización del conocimiento.

- $\quad$ La fortaleza de las universidades en el terreno de las TIC en la docencia está, como se dijo, en su profesorado y en el conocimiento. Pero ninguna universidad, como se dijo, es fuerte en todos los campos. Se ve imprescindible la alianza entre las instituciones, la formación de equipos interdisiciplinares, interinstitucionales que abarquen todos los aspectos necesarios para la calidad de los programas (técnico, pedagógico, comunicativo, etc.).

- Algunas cuestiones éticas de la cooperación.

- Cuando se plantean proyectos internacionales de explotación de las TIC en la docencia, lo más obvio suele ser ganar o ahorrar dinero. Dado que el costo de desarrollar un curso es fijo, ofrecer los cursos fuera de la propia jurisdicción permite una economía de escala. Es aquí, sin embargo, donde comienzan a presentarse algunos temas éticos. ¿Deben las instituciones en los países ricos con medios para desarrollar y distribuir programas de educación a distancia en los países pobres tratar de subsidiar sus programas a expensas de los países pobres? ¿Constituye la educación otra mercancía o servicio a ser vendido fuera? 
- $\quad$ Por otra parte, si los estudiantes en los países pobres quieren acceder a cursos de las más ricas y quizás más prestigiosas instituciones de fuera de sus propios países, y por lo tanto tener la oportunidad de mejores trabajos y mayor prosperidad, ¿Por qué vamos a evitar hacerlo?¿̇Qué implicaciones tendrá en la fuga de cerebros?

- La identidad cultural como elemento clave frente a la homogeneización cultural.

- La globalización de la educación superior implica un supranacionalismo que sobrepasa los límites nacionales, ignorando frecuentemente la identidad cultural. La tecnología con frecuencia va de la mano de esta globalización, aumentando la amenaza. Un asunto emergente en este contexto es, por ejemplo, el potencial de los sistemas de acreditación internacional. El incremento de la movilidad del capital humano, demanda reconocimiento mutuo y la globalización de la educación superior podría plantear amenazas a la acreditación de la educación superior de los sistemas nacionales. El sistema de acreditación internacional mismo supera los límites nacionales y podría implicar imperialismo cultural.

- En contraste con la globalización, la internacionalización de la educación superior -tal como la definimos al principio- reconoce las naciones y describe un proceso de intercambio de la educación superior entre naciones y/o instituciones. Esto implica asociación, entre naciones, entre sistemas nacionales, entre sistemas de acreditación, entre instituciones. Sin asociación, no hay educación internacional. Y las TIC tienen el potencial de reducir el costo de la distribución de esta educación.

\section{REFERENCIAS BIBLIOGRÁFICAS}

Bates, A. (1999). Cultural and ethical issues in international distance education Collaboration and Partnership in Distance Education UBC/CREAD conference, Vancouver, Canada, 2123rd, Sept. 1999.

Bricall, J. M. (1997). La universidad, al final del milenio. Conferencia de la CRUE "Los objetivos de la Universidad ante el nuevo siglo". Universidad de
Salamanca, 17 y 18 de noviembre de 1997.

Daniel, J. (1997). Distance Learning and the growth of the Mega-University. Hong Kong's First International Forum for World Leaders in Higher Education, 23 july. [en línea] Disponible en: http:// www.cityu.edu.hk/forum/pap1-5.html [consulta 2006, 18 de diciembre] 
Didou, S. (2005). Internacionalización y Proveedores externos de educación superior en América Latina y el Caribe. México- Caracas: ANUIES- IESALC.

Didou, S. (2006). Internacionalización de la educación superior y provisión transnacional de servicios educativos en América Latina: del voluntarismo a las elecciones estratégicas, en: IESALC: Informe sobre la Educación Superior en América Latina y el Caribe 20002005. La metamorfosis de la educación superior. Caracas, Venezuela: IESALC, 21-32.

Facundo, A. H. (2004). Tecnologías de la Información y Comunicación y Educación Superior Virtual en Latinoamérica y el Caribe. Evolución, Características y Perspectivas. Caracas, Venezuela: IESALC.

Harasim, L.; Hiltz, S.; Teles, L.; Turoff, M. (1995). Learning Networks. The MIT Press, Cambridge, MA.

IAU (2005). Sharing Quality Higher Education Across Borders: A Statement on Behalf of Higher Education Institutions Worldwide. International Association of Universities. [en línea] Disponible en: http://www.unesco. org/iau/p statements/index.html [consulta 2007, 28 de abril]

IIE (2006). Report on International Educational Exchange. Institute of International Education. [en línea] Disponible en: http://opendoors. iienetwork.org/ [consulta 2007, 28 de abril]

IIE (2007). Atlas of International Student Mobility. Institute of International Education. [en línea] Disponible en: http://www.atlas.iienetwork.org [consulta 2007, 28 de abril].

Roberts, T.; Romm, C.; Jones, D. (2000). Four Models of Online Learning, Proceedings of TEND-2000, Abu Dhabi, April 2000.
Salinas, J. (1995). Organización escolar y redes: Los nuevos escenarios de aprendizaje, en: Cabero, J.; Martínez, F. (Coord.): Nuevos canales de comunicación en la enseñanza. Madrid: Centro de Estudios Ramon Areces, 89118.

Salinas, J. (1996). Campus electrónicos y redes de aprendizaje, en: Salinas, J. y otros (Coord.): Redes de comunicación, redes de aprendizaje. Palma de Mallorca: Servicio de Publicaciones de la Universidad de las Islas Baleares, 91100. [en línea] Disponible en: http:// www.uib.es/depart/gte/salinas.html [consulta 2006, 18 de diciembre]

Salinas, J. (1997). Enseñanza flexible, aprendizaje abierto. Las redes como herramientas para la formación. Edutec'97. Creación de Materiales para la Innovación Educativa con Nuevas Tecnologías. Málaga [en línea] Disponible en: http://www.ieev.uma. es/edutec97/edug7 c1/2-1-00.htm [consulta 2006, 18 de diciembre]

Salinas, J. (1998). Redes y Educación: Tendencias en educación flexible y a distancia, en: Pérez, R. y otros: Educación y tecnologías de la educación. II Congreso Internacional de Comunicación, tecnología y educación. Oviedo. 141-151 [en línea] Disponible en: http://www.uib.es/ depart/gte/tendencias.html [consulta 2006, 18 de diciembre]

Salinas, J. (1999). Un modelo de formación flexible en entornos virtuales para la discusión. II Jornadas de Tecnologías de la Información y Comunicación en las Universidades Españolas. CRUE Universidad de Zaragoza.

Salinas, J. (2001). Universidades Globales Multinacionales: Redes de aprendizaje $\mathrm{y}$ consorcios institucionales para el desarrollo de la educación flexible, en: Salinas, J.; Batista, A. (Coord.): Didáctica y Tecnología Educativa para 
una universidad en un mundo digital. Universidad de Panamá: Imprenta Universitaria, 42-65.

Salinas, J. (2002). Modelos flexibles como respuesta de las universidades a la sociedad de la información. Acción Pedagógica 11(1), enero-junio.

Salinas, J. (2004). Cambios metodológicos con las TIC. Estrategias didácticas $\mathrm{y}$ entornos virtuales de enseñanzaaprendizaje. Bordón 56 (3-4).

Silvio, J. (2000). La virtualización de la universidad ¿́cómo transformar la educación superior con la tecnología? Caracas, Venezuela: IESALC. UNESCO.
Silvio,J.(2003).TendenciasdelaEducación Superior Virtual en América Latina y el Caribe. Caracas, Venezuela: IESALC. UNESCO.

Trenchs, M. Et alt. (2004). La consolidación de un proceso de colaboración interunivesitaria de docencia virtual: el proyecto intercampus en cataluña. EDUTEC'2004, Barcelona. [en línea] Disponible en: http://edutec2004.lmi. ub.es/pdf/55.pdf [consulta 2007, 28 de abril]

\section{PERFIL ACADÉMICO Y PROFESIONAL DEL AUTOR}

Jesús Salinas. Doctor en Ciencias de la Educación. Profesor de Tecnología Educativa en la Universidad de las Islas Baleares. Coordinador del Curso de Doctorado Interuniversitario en Tecnología Educativa. Investigador principal del Grupo de Tecnología Educativa. Director de Edutec. Revista electrónica de Tecnología Educativa.

E-mail: Jesus.salinas@uib.es

DIRECCIÓN DEL AUTOR

Dep. de Ciencias de la Educación.

Universidad de las Islas Baleares

Cra. de Valldemossa Km 7,5 07122

Palma de Mallorca, España

Fecha de recepción del artículo: 11/06/07

Fecha de aceptación del artículo: 17/09/07 
